\title{
Defining Training and Performance Caliber: A Participant Classification Framework
}

\author{
Alannah K.A. McKay, Trent Stellingwerff, Ella S. Smith, David T. Martin, Iñigo Mujika, \\ Vicky L. Goosey-Tolfrey, Jeremy Sheppard, and Louise M. Burke
}

\begin{abstract}
Throughout the sport-science and sports-medicine literature, the term "elite" subjects might be one of the most overused and illdefined terms. Currently, there is no common perspective or terminology to characterize the caliber and training status of an individual or cohort. This paper presents a 6-tiered Participant Classification Framework whereby all individuals across a spectrum of exercise backgrounds and athletic abilities can be classified. The Participant Classification Framework uses training volume and performance metrics to classify a participant to one of the following: Tier 0: Sedentary; Tier 1: Recreationally Active; Tier 2: Trained/Developmental; Tier 3: Highly Trained/National Level; Tier 4: Elite/International Level; or Tier 5: World Class. We suggest the Participant Classification Framework can be used to classify participants both prospectively (as part of study participant recruitment) and retrospectively (during systematic reviews and/or meta-analyses). Discussion around how the Participant Classification Framework can be tailored toward different sports, athletes, and/or events has occurred, and sportspecific examples provided. Additional nuances such as depth of sport participation, nationality differences, and gender parity within a sport are all discussed. Finally, chronological age with reference to the junior and masters athlete, as well as the Paralympic athlete, and their inclusion within the Participant Classification Framework has also been considered. It is our intention that this framework be widely implemented to systematically classify participants in research featuring exercise, sport, performance, health, and/or fitness outcomes going forward, providing the much-needed uniformity to classification practices.
\end{abstract}

Keywords: elite, athlete, terminology, classification system, exercise, recreationally active

The physiology and performance of athletes are thoroughly scrutinized by many audiences, including sport and exercise scientists who are interested in the extremes of human performance, the contributions of training and genetics to sport performance, the impact of various strategies or interventions, and the potential lessons that could be inferred for the benefit of the general community. However, the sports science and sports medicine literature, from elite athletes through to sedentary individuals, has evolved over time without a common perspective or terminology to characterize the caliber and training status of a participating individual or group of individuals. Indeed, the term "elite" subjects might be one of the more overused and ill-defined terms in the exercise science literature. ${ }^{1}$ An investigation examining 91 publications, which defined their participants as either "elite" or "expert" across a range of sports, found that the range of this term encompassed athletes participating in national-level competitions up to athletes winning medals at major international

McKay, Smith, and Burke are with the Mary MacKillop Inst for Health Research, and Martin, the School of Behavioural and Health Sciences, Australian Catholic University, Melbourne, VIC, Australia. Stellingwerff is with the Inst for Sport Excellence, Canadian Sport Inst Pacific, Victoria, BC, Canada. Stellingwerff and Sheppard are with Exercise Science, Physical \& Health Education, University of Victoria, Victoria, BC, Canada. Mujika is with the Dept of Physiology, Faculty of Medicine and Nursing, University of the Basque Country, Leioa, Basque Country, Spain, and the Exercise Science Laboratory, School of Kinesiology, Faculty of Medicine, Universidad Finis Terrae, Santiago, Chile. Goosey-Tolfrey is with the Peter Harrison Centre for Disability Sport, School of Sport Exercise and Health Sciences, Loughborough University, Loughborough, United Kingdom. Sheppard is also with Canada Snowboard, Whistler, BC, Canada. McKay (alannah.mckay@acu. edu.au) is corresponding author. competition. ${ }^{2}$ Terms such as "trained," "highly trained," and "well-trained" also seem to be highly subjective, with variations around the participants training load, training history, and the intent of the training commitment. 3,4

There are many benefits to having a standardized framework to characterize participants based on an integrated approach to their sporting performance/achievements, biometric attributes, training exposure, and general fitness level. For example, the basic principle of the specificity of research, which states that the results of a study should be applied only to populations and/or scenarios which are similar to those involved in the research design, ${ }^{5}$ requires objective features to define the targeted individuals/groups. Comparisons across and within studies, which try to identify the similarities or differences between members of a sporting or active population, require robust definitions of the cohort. There is evidence that athletic success might be explained by different attributes, or that athletic caliber may influence the response to various interventions. For example, although targeted periodization of carbohydrate availability has been shown to enhance training adaptation and endurance performance in subjects described as "trained athletes," ${ }^{6,7}$ these benefits have not been seen when similar interventions were implemented in "elite" and "highly trained" athletes. ${ }^{8-10}$ Similarly, beetroot juice/nitrate is considered an evidence-based performance enhancing supplement, ${ }^{11}$ based largely on studies in lower-caliber athletes, whereas elite athletes do not appear to obtain the same benefits. ${ }^{12,13}$ Whether these and other observations are robust and can be explained by innate biometric differences or by the modifying effects of training history and load are of particular interest. However, the results of existing and future studies cannot be effectively harvested unless there is uniformity around the descriptive characteristics and classification of athletic populations. 
Early attempts to define an elite performer were based on time spent practicing a task, with suggestions that 10 years ${ }^{14}$ or 10,000 hours ${ }^{15}$ are required to achieve expert level. However, such a model would imply that anybody can reach the elite level with deliberate practice and intent, whereas in an elite sporting context other factors such as biological age ${ }^{16}$ and genetics ${ }^{17}$ are undoubtedly important. Additionally, time-based metrics of training are unlikely to be comparable across sports due to the nature of training; for example, a sprinter will most likely require fewer training hours compared to an endurance cyclist to reach the elite level. Furthermore, there are numerous examples of action sports athletes who have transferred skills gained in one domain to an entirely different domain ${ }^{18}$ and have surpassed the top competitors in the sport who have had $>10,000$ hours of training.

Specific attempts to classify the training and performance caliber of athletes have occurred. Two decades ago, Jeukendrup et $\mathrm{al}^{19}$ recognized the misclassification of training status in cyclists throughout the literature, and attempted to unify classification and terminology based on metrics of training and race status (training volume, training background, and race days per year), combined with quantitative physiological variables (measures of aerobic capacity, power output, and efficacy). More recently, 2 reviews further developed cycling-based classification protocols, terminology, and performance indicators in both male 4 and female participants. ${ }^{3}$ These frameworks consist of 5 performance levels, which encompass untrained through to professional cyclists, and purposely use neutral terminology to avoid the misinterpretation of the commonly used subjective terms such as "well-trained" or "elite." Within the cycling specific literature, these classification systems have been widely adopted and have likely facilitated better comparison and interpretation of scientific results. However, these classification systems are highly specific to a single sport and rely on extensive physiological and anthropometric data for classification to occur.

The most extensive attempt at defining the "elite" athlete was completed in 2015, following an examination of the misuse of the term in sports psychology research. ${ }^{2}$ Swann and colleagues proposed that 5 criteria should be used to assess an athlete's ability: (1) highest performance standard, (2) level of success, (3) years of experience, (4) competitiveness of the sport within the athlete's country, and (5) global competitiveness of the sport, with responses used in a mathematical equation to differentiate a "semi-elite" athlete from a "world-class" athlete. However, this has yet to be established within the exercise sciences/physiology domain, and the rigidity of classification does not consider the many nuances that are apparent between different sports and athletes.

The current paper builds upon this previous literature and presents a flexible framework that aims to:

1. Provide a training and performance-based classification system grounded in population statistics, meaning only a tiny proportion of participants can reach the top tiered classification level as in elite sport, with the vast majority of the population being either sedentary or recreationally active;

2. Develop a continuum whereby all participants can be classified across a spectrum of exercise backgrounds and athletic abilities, from sedentary individuals through to world-class athletes;

3. Develop a framework that can be implemented both prospectively (ie, as part of study participant recruitment to publication) and retrospectively (ie, during systematic reviews and/or meta-analyses) to classify participants;
4. Allow classification to occur without the need for extensive physiological, anthropometric, biomechanical, or skill-based testing;

5. Be a user-friendly resource, which can be implemented with research participants across the spectrum of training status/ sports and across all scientific disciplines;

6. Provide a flexible framework that endures the test of time, which can eventually be adapted as sports and athletic ability inevitably evolves over time.

It is our intention that this framework should be widely implemented to systematically classify participants in research and published articles featuring exercise, sport, performance, health, and/or fitness outcomes going forward.

\section{A New Paradigm for Participant Training and Performance Classification}

A framework, presented in Table 1, has been developed by a range of content/discipline experts to facilitate both prospective and retrospective classification of individual participants in studies featuring exercise/training and/or sport performance outcomes. Each author has extensive expertise working in elite sporting environments, along with extensive academic experience, which allowed the construction of a holistic framework whereby individuals are classified based on training and performance metrics into 6 possible tiers. Furthermore, we have extended the framework by estimating the percentage of the population which might fall into each classification. These population estimates have been based on both global and continent-specific statistics to demonstrate how densely populated each of these tiers may be, also allowing authors an opportunity to reflect on how well the framework represents real-life scenarios. We have provided calculations and appropriate references for these statistics within the Supplementary Material (available online).

The first, most populated tier in this framework (Tier 0: Sedentary) includes individuals who fail to meet the World Health Organization's physical activity standards for adults: $>150$ minutes of moderate activity or $>75$ minutes of vigorous activity each week. ${ }^{20}$ Meanwhile, Tier 1: Recreationally Active involves individuals who achieve the physical activity standards ${ }^{20}$ and may participate in a variety of activities and/or sports without a specific commitment or focus on competition. Several country estimates suggest that between $48 \%$ and $54 \%$ of individuals do meet current physical activity guidelines, ${ }^{21-24}$ which would classify them as Recreationally Active or above, according to our framework. Participants in Tiers 0 or 1 can contribute valuable information to the sports science literature by forming suitable control groups in cross-sectional/observational studies of athletes, or as the subject of mechanistic-based studies, including pharmacokinetic, dose-response, and proof-of-concept investigations. However, their inclusion as participants in investigations of sports performance is inappropriate due to the poor reliability of performance measures collected on these populations and because these cohorts have little validity compared to higher tiers.

Tier 2: Trained/Developmental is estimated from national reports of sport participation rates to include $\sim 12 \%$ to $19 \%$ of the population. ${ }^{25-27}$ Individuals in this tier identify with a given sport and are differentiated from Tier 1 because of their commitment to sports-specific training $\sim 3$ times per week and an intention to compete in local-level competitions. No performance criteria or skill level is required to reach this classification. Individuals in this tier represent a relatively large percentage of the available 


\section{Table 1 Participant Classification Framework}

\begin{tabular}{l} 
Tier \\
\hline Tier 5: World Class \\
$=<0.00006 \%$ of the global population \\
$=<0.001 \%$ of the Australian population
\end{tabular}

Tier 4: Elite/International Level

$=\sim 0.0025 \%$ of the global population $=\sim 0.0055 \%$ of the Australian population

Tier 3: Highly Trained/National Level

(Provincial/State or Academy Programs) $=\sim 0.014 \%$ of the global population

$=\sim 0.027 \%$ of the Australian population

\section{Tier 2: Trained/Developmental $=\sim 12 \%-19 \%$ of the global population $=\sim 18 \%$ of the Australian population}

\section{Tier 1: Recreationally Active} $=-35 \%-42 \%$ of the global population $=\sim 30 \%$ of the Australian population

\section{Tier 0: Sedentary}

$=\sim 46 \%$ of the global population $=\sim 52 \%$ of the Australian population

\section{Criteria for classification}

- Olympic and/or world medalists.

- World-record holders and athletes achieving within $2 \%$ of world-record performance and/or worldleading performance.

- Top 3-20 in world rankings and/or top 3-10 at an Olympics/World Championships (ie, finalists in their event), with this number determined based on size and depth of competition in the event.

- Top players within top teams (teams which medal or are in the most competitive leagues) or athletes achieving individual accolades (ie, most valuable player, player of the year).

- Maximal, or nearly maximal training, within the given sports norms.

- Exceptional skill-level achieved (ie, running biomechanics, ball skills, acquired decision-making components).

- Competing at the international level (individuals or team-sport athletes on a national team).

- Team-sport athletes competing in international leagues/tournaments.

- Top 4-300 in world rankings, with this number dependant on size and depth of competition in the event.

- Achievement of within 7\% of world-record performance and/or world-leading performance.

- NCAA Division I athletes.

- Maximal, or nearly maximal training, within the given sports norms, with intention to complete at top-level competition.

- Highly proficient in skills required to perform sport (ie, biomechanics, ball skills, acquired decisionmaking components).

- Competing at the national level.

- Team-sport athletes competing in national and/or state leagues/tournaments.

- Achievement of within $\sim 20 \%$ of world-record performance and/or world-leading performance.

- NCAA Division II and III athletes.

- Completing structured and periodized training and developing towards (within 20\%) of maximal or nearly maximal norms within the given sport.

- Developing proficiency in skills required to perform sport (ie, biomechanics, ball skills, acquired decision-making components).

- Local-level representation.

- Regularly training $\sim 3$ times per week.

- Identify with a specific sport.

- Training with a purpose to compete.

- Limited skill development.

- Meet World Health Organization minimum activity guidelines: Adults aged 18-64 years old completing at least 150 to $300 \mathrm{~min}$ moderate-intensity activity or 75-150 min of vigorous-intensity activity a week, plus muscle-strengthening activities 2 or more days a week. ${ }^{20}$

- May participate in multiple sports/forms of activity.

- Do not meet minimum activity guidelines.

- Occasional and/or incidental physical activity (eg, walking to work, household activities).

Abbreviation: NCAA, National Collegiate Athletic Association. Note: We have presented the key classification criteria for each tier and the estimated proportion of the population that fall into each tier. These population estimates have been derived from both global and continent-specific statistics to demonstrate how densely populated each of these tiers may be. Detailed calculations for these estimates can be found in the Supplementary Material (available online).

population of trained individuals and are well suited for trials that require large subject numbers to be adequately statistically powered. This may include cross-sectional, observational, or surveybased study designs. When Tier 2 participants are involved in performance-based research, we recommend the use of familiarization trials and established and sensitive laboratory-based tests with a known typical error. This will reduce the noise-to-signal ratio and allow the best chance at detecting changes in performance in this population. ${ }^{28}$

Tier 3: Highly Trained/National Level is the first tier of the present framework in which performance standards are defined and represent only $\sim 0.01 \%$ to $0.03 \%$ of the population (see Supplementary Material [available online]). Athletes within this tier include national-level representatives who are performing structured and/or periodized training and are developing proficiency in skills required to perform their sport at the highest level. This tier includes individuals participating in Provincial/State or Academy Programs (eg, Division II and III programs of the National Collegiate Athletic Association). Tier 3 athletes can be expected to provide reliable measures of performance in familiar tasks and are therefore well suited to both laboratory- and fieldbased research. Since individuals from higher tiers are often reluctant to participate in studies using invasive measurements or requiring large time commitment, Tier 3 athletes often represent a "sweet spot" in studies that simultaneously collect mechanistic data with reliable performance outcomes. 
Tier 4: Elite/International Level includes $\sim 0.003 \%$ to $0.006 \%$ of the population based on our estimates of participation rates in international-level competitions (see Supplementary Material [available online]). This classification requires a higher performance level than Tier 3 and is likely to involve representation at international-level competition. Athletes within Tier 4 may perform within $\sim 7 \%$ of worldleading or world-record performance, be ranked within the world's top 4-300 athletes (depending on the depth of field within the sport), and/ or compete in professional leagues/tournaments. The majority of individuals at this level are a part of the registered testing pool or national testing pool as defined by World Anti-Doping Agency. ${ }^{29}$ Conducting studies in Tier 4 athletes can be challenging due to their smaller numbers and availability. Nevertheless, specialized research projects involving individuals at this level can provide particularly valuable insights and outcomes with high ecological validity, which can often be directly translated into high-performance sport settings.

Finally, Tier 5: World Class is a classification reserved for the most exceptional athletes within their sport/event. This includes Olympic and/or World Championship medalists and finalists, top athletes within top global teams, and athletes who can perform within $\sim 2 \%$ of worldleading and/or world-record performances. Tier 5 athletes are considered the "crème of the crop," and their rarity makes an involvement in cohort studies to be highly unusual. Indeed, case studies are the more common research design to feature these athletes, yielding valuable information about the top-end of athletic performance..$^{30-32}$

In Figure 1, we show the interaction between these classification tiers, training volume/physical activity level, performance standards, research design, and the population density of each tier.

\section{How to Use the Participant Classification Framework}

\section{Prospectively}

Figure 2 provides a flow chart that demonstrates how information should be collected and interpreted in a stepwise manner. It should

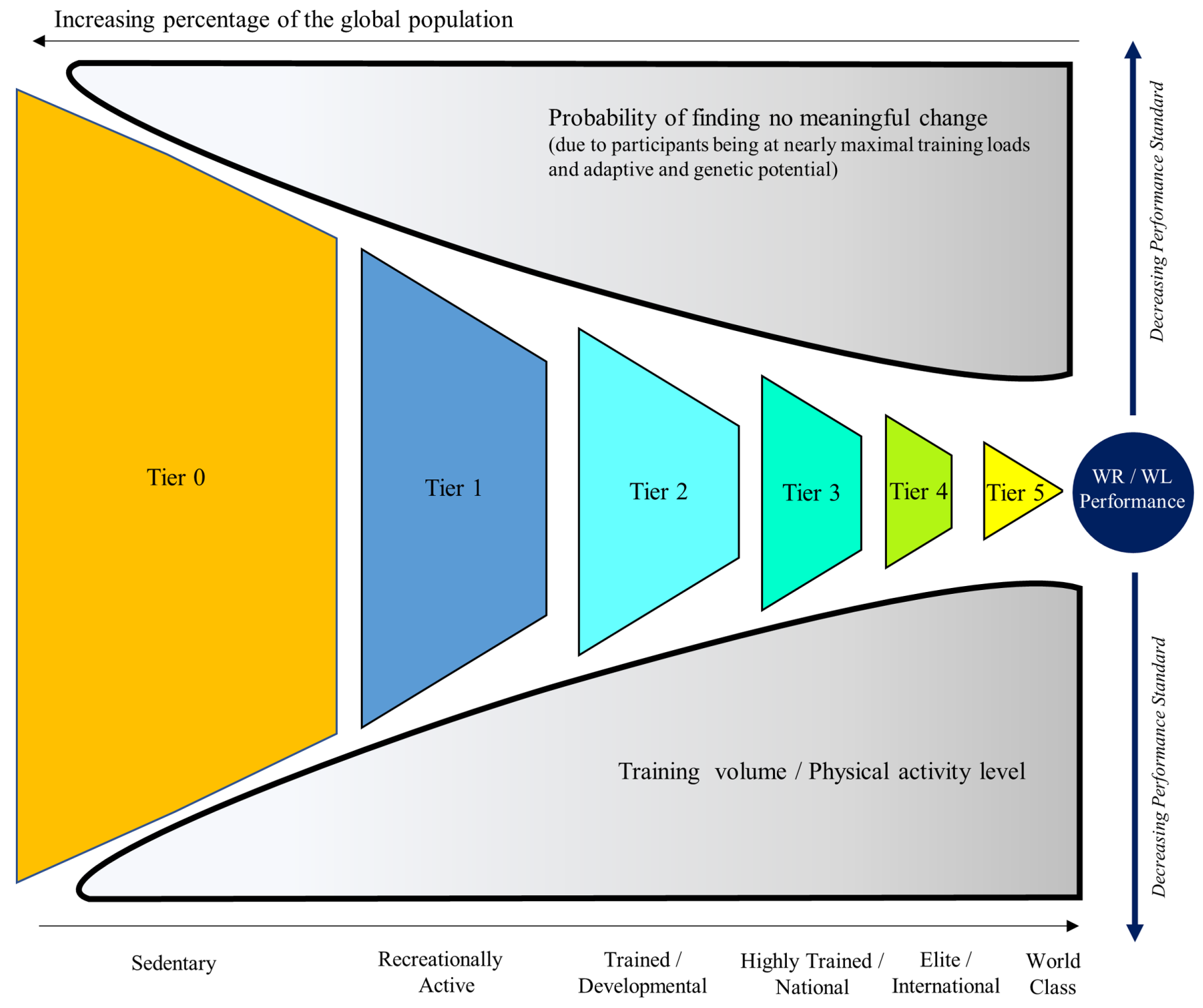

Figure 1 - Graphical representation of the interaction between the Participant Classification Framework, training volume/physical activity levels, population proportions, and research design. Inspired by D. Lee Hamilton. ${ }^{33}$ WL indicates world leading; WR, world record. 


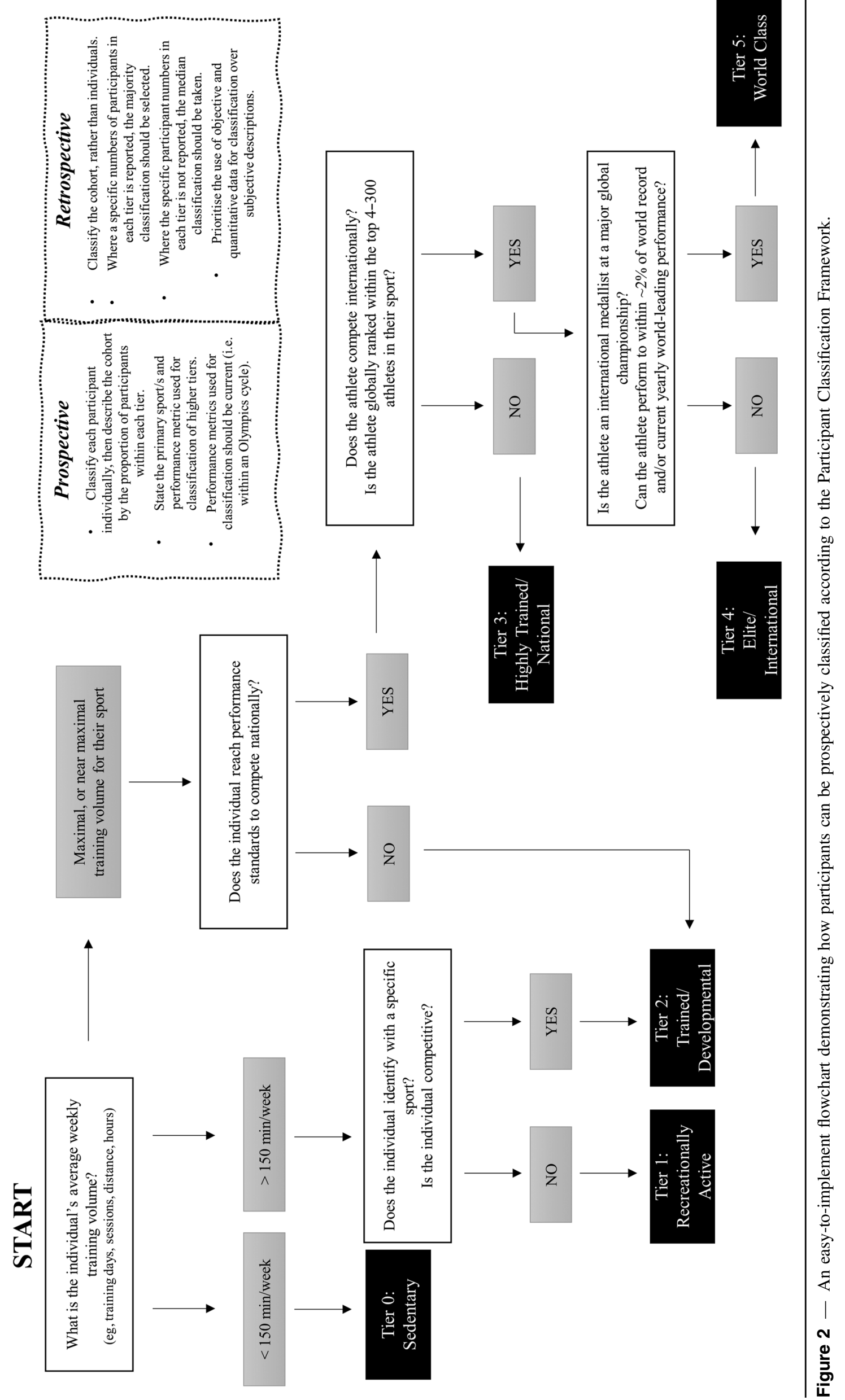


be noted that individuals are not required to meet all aspects of each tier to be classified, rather the information provided in Table 1 should be used in conjunction with the process outlined in Figure 2 to guide classification. When participants are recruited, average exercise volume is the first characteristic that should be identified; this can be determined from common metrics such as the number of training days per week, training hours, and quantification of tasks that are completed (eg, kilometers traveled or number of throws/ jumps per week). The chosen metric should then be scaled according to considerations of normal preparation for a world-class athlete in the given sport. This will vary markedly between sports; for example, a world-class road cyclist may train in excess of $1000 \mathrm{~km} /$ wk $(>30 \mathrm{~h})$, whereas some middle-distance runners of this caliber may only run $\sim 50 \mathrm{~km} / \mathrm{wk}$ (approximately $4-5 \mathrm{~h}$ ). This information should provide a clear distinction between Tier 0: Sedentary Individuals (minimal training) and those in Tier 3: Highly Trained/National Level or above (near maximal training volume for their chosen sport). Differentiation between Tier 1: Recreationally Active and Tier 2: Trained/Developmental individuals requires a greater understanding of their primary training modality and recent competition history, with identification with a specific sport, and an intention to compete being the primary factor splitting these 2 tiers. Differentiation of the top 3 athletic tiers requires an understanding of the athlete's performance achievements. This information can be collected from personal best times/performance, world rankings, participation in major competitions, or achievement of individual athletic accolades (eg, medals, world records, athletic-based awards), which should be relevant to the athlete's primary sport. We suggest that performance metrics used for this assessment be current: within a 4-year Olympic cycle or an appropriate sport-specific time frame for non-Olympic sports (eg, between seasons or world championships). This allows current performances to be considered for classification, rather than a previous performance standard that is unlikely to be achieved again. It should be noted that the suggested performance metrics are examples only, and this framework has been intentionally created to allow for appropriate sport specific metrics to be used.

To ensure a consistent and informative approach to documenting a participant's training and performance caliber, we have outlined simple reporting guidelines that should be adopted when using this framework. First, it is suggested that authors classify each participant individually during study recruitment, and then describe the cohort by reporting the proportion of participants within each tier. This avoids generalizations or embellishment of a cohort's athletic ability. Furthermore, all studies should also report the sex of the participant cohort, or the male-tofemale ratio in mixed sex studies. In studies utilizing athletes (Tiers 3-5), the primary sport(s) should be explicitly mentioned. While not compulsory, we recommend that the key performance-based metrics used to classify individuals also be reported, to allow the reader greater context for interpretation. Similarly, other sportspecific details regarding competition experience, position within a team or event within a sport, and previous years' experience all may be important when describing athletes from higher tiers.

A final consideration, which is not currently accounted for within the framework, is the fitness status at the time of testing, relative to an individual's classification according to the framework. For instance, a current Olympic medalist who is returning from injury at the time of classification would still be considered Tier 5: World Class, based on training volume and previous performance outcomes. However, their performance capabilities at the time of their involvement in a study may not reflect their classification. Conversely, an emerging athlete who has been classified as Tier 3: Highly Trained/National Level but has had limited racing opportunities may outperform athletes at Tier 4: Elite/International Level. These complexities are nuanced and will be highly specific to each individual's situation. Accordingly, we encourage reporting additional descriptive information relating to the current training status of individuals where appropriate. This may include the participant's training phase (eg, off-season, preseason, competition phase); training status (eg, full training, modified training); and any other relevant information (ie, recent career interruptions, competition history).

\section{Retrospectively}

We envisage that this framework can also be used in a retrospective manner to classify participant cohorts for the purpose of audits, reviews, and/or meta-analyses. In this instance, subject characteristics (means with SDs) which are reported alongside other descriptive information should be interpreted using the framework to provide an overview of the caliber of the study cohort. Identifying with a particular sport typically differentiates Tier 2: Trained/Developmental from Tier 1: Recreationally Active individuals, while studies of Tier 2: Trained/Developmental individuals often also provide a sport-specific metric of training volume (eg, kilometers per week, rather than days/week, which is more common with Tier 1: Recreationally Active individuals). Typically, studies which include higher caliber athletes (Tier 3-5) provide a greater depth and breadth of information regarding participant training status. Importantly, objective information should be prioritized for the purposes of classification, even if subjective terminology employed by authors contradicts the resulting classification tier. For example, studies describing "recreationally active" participants with an activity level less than 150 minutes per week should be classified as Tier 0: Sedentary. Furthermore, participants who are described as competing at an international level but present performance metrics that are far from the required performance level should be classified as Tier 3: Highly Trained/National rather than Tier 4: Elite/International. In other words, objective and quantitative data should be prioritized above prior published subjective descriptions.

While we have encouraged a specific approach to the prospective reporting of study participants, when the framework is used to retrospectively describe study cohorts, a classification of the typical member or majority of the participant group is likely to be the most practical outcome. Similarly, where appropriate, studies conducted on a sporting team should classify the group as a whole rather than differentiating team members. Since the type of information typically provided to make specific or individual assessments is likely to be missing, a consistent and wide-reaching approach to classifying individuals is therefore preferred. To determine the "majority" classification when information around the proportion of participants in each tier is not provided, the median tier should be chosen. For example, if participants ranged between Tier 2 and Tier 4, the study should be classified as Tier 3 . In scenarios where participants range between 2 tiers, the higher tier should be selected (eg, Highly Trained/National and Elite/ International Level individuals would be classified as Elite/International level). When studies are intentionally designed to compare individuals of different training status, each cohort should be classified as a separate group. Undoubtedly, some studies will fail to provide clear information about their participants, preventing a robust decision around classification to occur; here, the study should be classified as "insufficient information." It should be 
noted that the retrospective use of this framework should not require studies to provide extensive information covering all criteria identified in the tables in this review. Rather, the available information in any published study should be used to provide the best-fit assessment of the caliber of the cohort involved. Table 2 provides examples of a random selection of papers from sport and exercise science journals, which have been retrospectively classified based on the proposed framework.

\section{Classifying Athletes Across Different Sports}

While the aim of this framework is to provide a resource that can be adapted to all athletes and research participants, we recognize there will be key differences between sports. In Table 3, we have clustered sports into 7 categories based on physiological demands, tactical components, and the skill requirements of the sport. Within each category, we have outlined considerations that should be used when applying the Participant Classification Framework to these sports. First, team sports are unique in the way participants are classified. According to Stefani's taxonomy of sports rating systems,${ }^{40}$ team sports can be described as "object sports" where the primary objective is to manipulate an object while another competitor is in direct confrontation. Due to the dynamic nature of these sports, using performance times or world-record standards for classification using the Participant Classification Framework is unsuitable, and therefore classification should occur primarily based on team representation and playing position. Table 4 provides a specific example of the application of the Participant Classification Framework to basketball. This suggests that Tier 5: World Class includes only the top players ("starters") from the top global league/tournaments, while the next 2 tiers include athletes playing in international leagues (Tier 4) and domestic/ state leagues (Tier 3).

The next 3 sports categories cover Endurance/Long-Distance sports, Middle-Distance/Power sports, and Speed/Strength sports. These sports are similar, with the majority being described as "independent sports" 40 in which one competitor cannot directly interfere with the performance of another. In these sports, performance can be quantified, allowing comparisons over time and across locations. In Table 5, we have used the Participant Classification Framework to generate benchmarked performance standards for selected events within track and field across Tiers 3 to 5. Here, we have derived performance standards using a number of different metrics, including world-leading times, world-record times, and the leading time at the most recent major international competition (Tokyo 2020 Olympic Games). We do not intend for this table to be used as a strict resource from which participants are classified. Rather, this has been included to demonstrate the numerous ways performance standards may be generated for use in the Participant Classification Framework, while also highlighting methods which may not be appropriate. For instance, the marathon at the Tokyo 2020 Olympic Games was performed in very hot and humid conditions $\left(\sim 28^{\circ} \mathrm{C}\right.$ and $\sim 70 \%$ humidity), ${ }^{47}$ leading to slower race times (winners finished in $\sim 106 \%$ and $110 \%$ of world-record time for males and females, respectively). Therefore, using these times to benchmark performance standards would not be appropriate. Similarly, the use of world records may not be the best option for benchmarking performance standards in some sports. An example of this is the men's long jump, in which the world record was set more than 30 years ago (1991: $8.95 \mathrm{~m}$ ) and only one other athlete has performed to within $2 \%$ of the world record since that time. ${ }^{43}$ When world records are dated or current performances are not close to these standards, world-leading performance is a far more appropriate metric from which to derive performance standards. While the majority of sports in these categories easily allow performance to be quantified in a standardized way, there are a few exceptions. For example, while rowing is a time-based independent sport, boat speed can be highly influenced by environmental factors such as water temperature, salinity, wind, and water current direction. Furthermore, sports such as BMX racing and Mountain Bike are performed on different courses with different features, and therefore absolute race time is not comparable across racing locations. In these instances, world rankings should be the primary metric used for classification.

The remaining three categories refer to precision/skill-dependent sports, racquet sports, and combat/weight-making sports. These include a mixture of object sports, independent sports, and combat sports in which a competitor tries to directly control the opponent by confrontation. ${ }^{40}$ These sports often have a larger dependence on skill rather than physiology or strength as a primary determinant of success, which can be factored into classification. In the case of sports which are subjectively scored, using performance standards may not be appropriate simply because scores allocated during the judging process are primarily intended to differentiate placing, rather than act as a highly sensitive instrument to reflect the specific magnitude of performance difference. Accordingly, the magnitude of score difference between 2 participants may be larger than the magnitude of actual technical difference. For this reason, sports from all 3 of these categories will likely rely heavily on world rankings for classification using the Participant Classification Framework. Often, points are awarded to athletes based on competition outcomes which contribute to their world ranking, with many sports adjusting the way points are obtained to prioritize key events. For example, the ranking method used by the Association of Tennis Professionals allocates a higher number of points according to the progression of the athlete within a tournament, with an increase in points distributed at Grand Slams and Association of Tennis Professional Finals, compared to less prestigious competitions. ${ }^{48} \mathrm{Simi}$ larly, the International Judo Federation also uses a points-based system, allocating points based on final competition standings, with increased points awarded at major-level competitions. ${ }^{49}$ In Table 6, we have provided an example for classification in the sport of snowboard, including specific skill-based metrics and world ranking brackets for each tier. Once appropriate metrics have been chosen to classify an individual, we foresee that 3 key factors will require further sport-specific consideration.

Depth of Participation in the Sport. It should be recognized that sports have different rates of global participation. For instance, running is relatively inexpensive and has minimal requirement for skill or equipment for participation at the recreational level, making it popular worldwide ( $>200$ countries represented at global championships). Conversely, sports such as Sailing, Equestrian, Modern Pentathlon, and Ski Jump all require expensive equipment, expert coaching, and/or the necessary climate for participation to occur. These barriers explain small global participation rates with a subsequent effect on the number of athletes who compete at the elite level. These sports often feature a small competitor pool with variable performance levels at major events. For instance, separation between first and eighth finisher in the $100 \mathrm{~m}$ women's freestyle swimming final at the Tokyo 2020 Olympic Games was 1.27 seconds or $2.4 \%$ of winning race time. ${ }^{50}$ Conversely, in the relatively new Olympic sport of women's park skateboard, the 22.8 point difference from first to eighth in the final equates to 


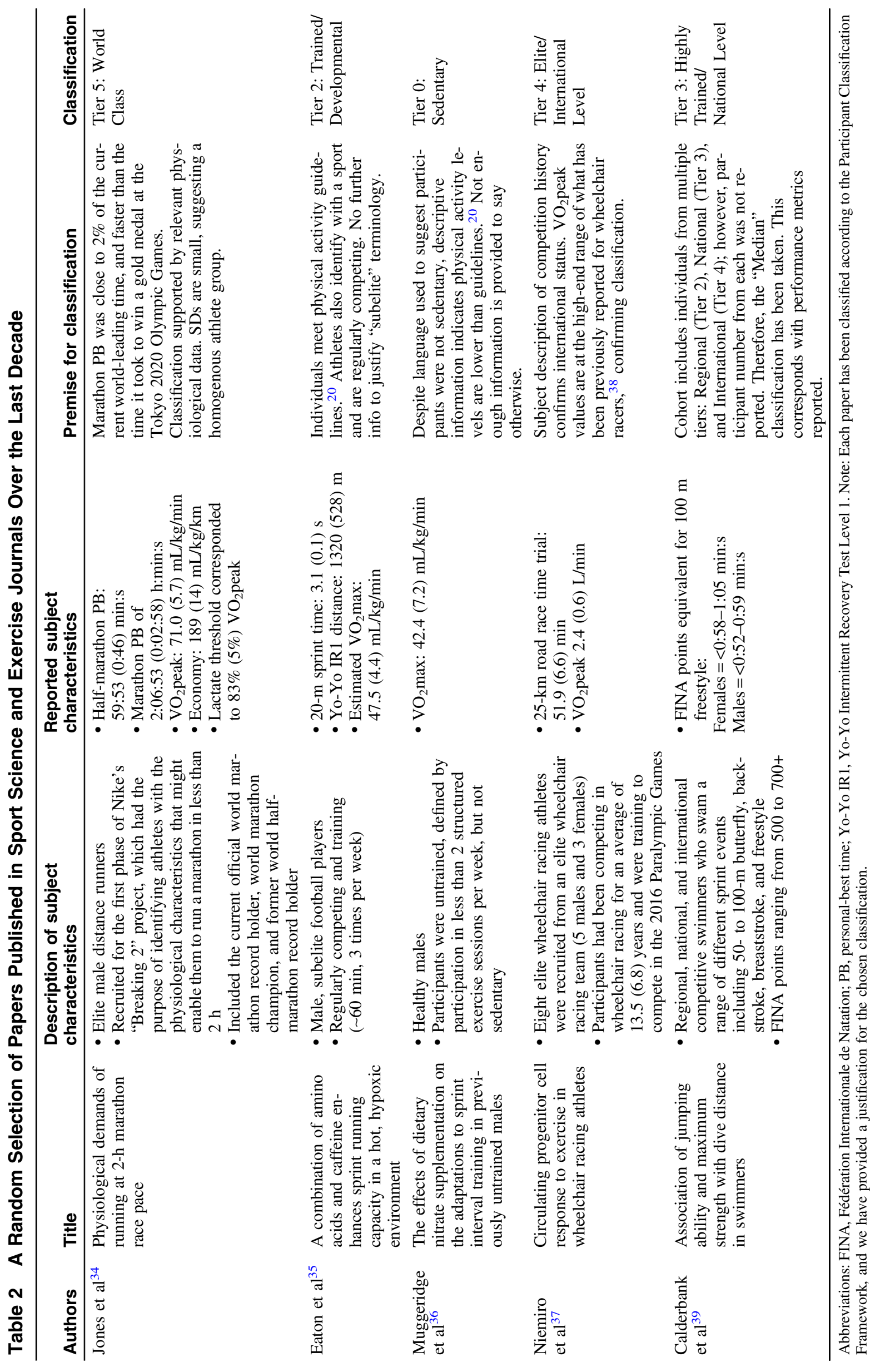




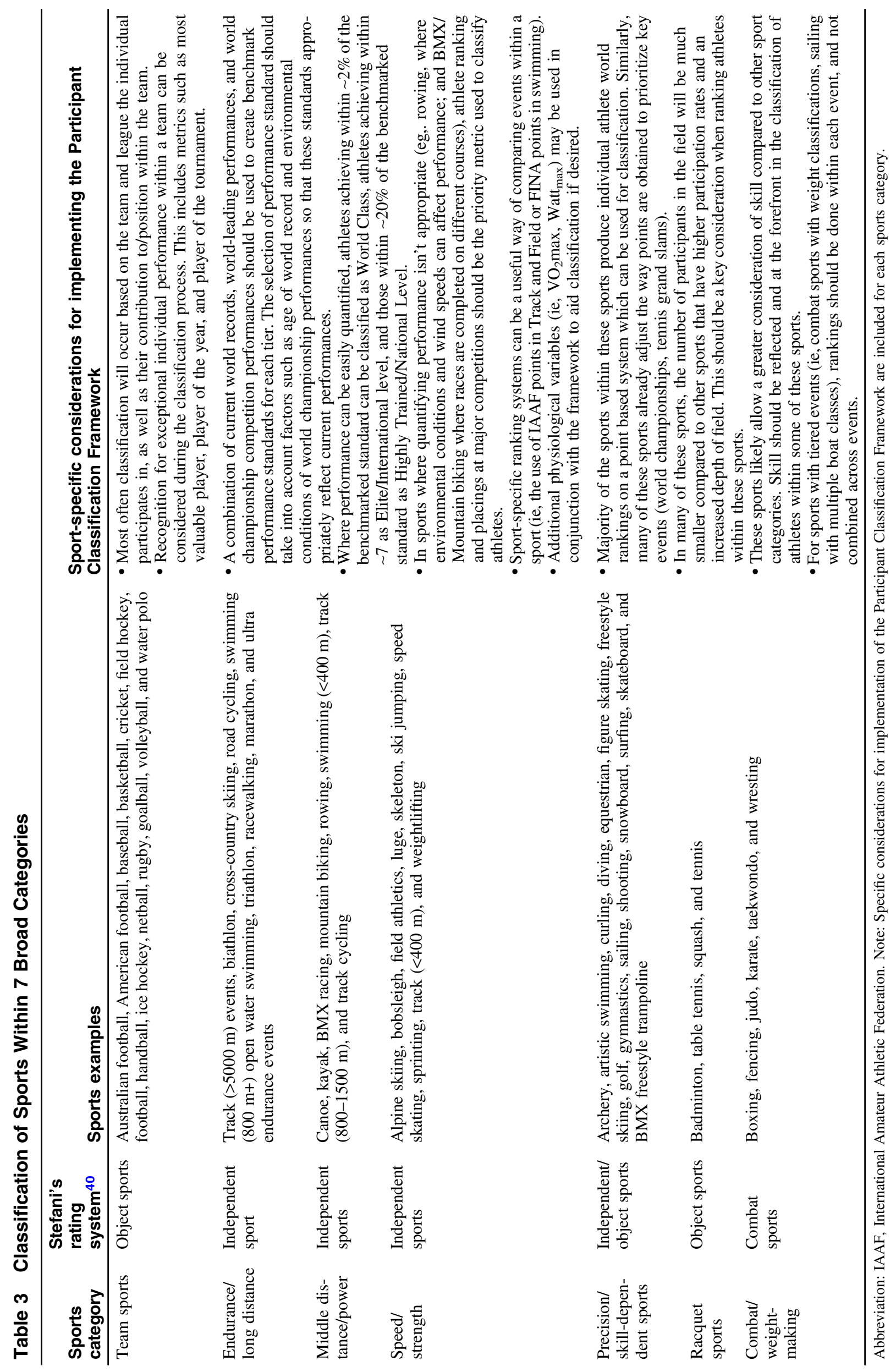




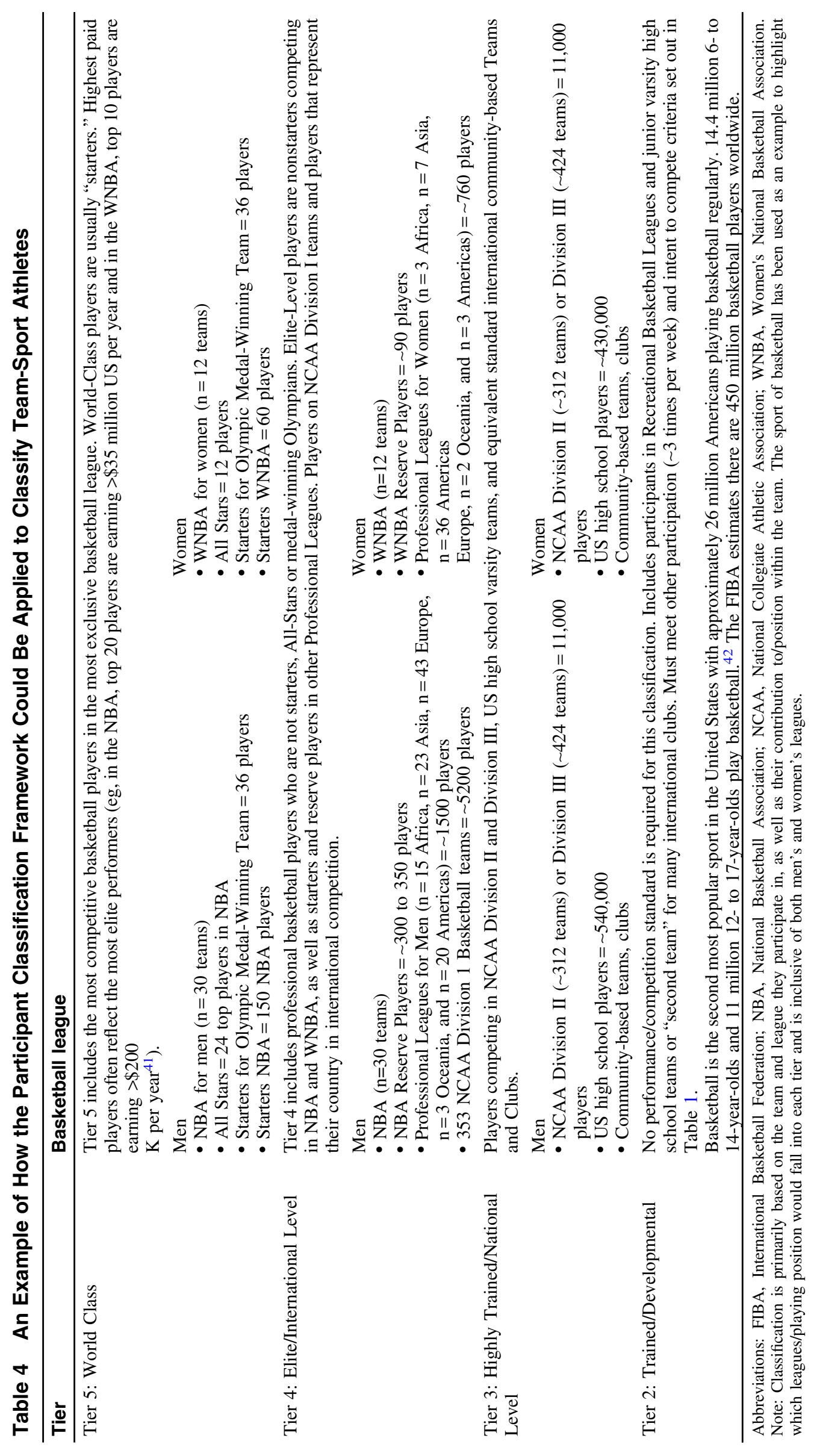




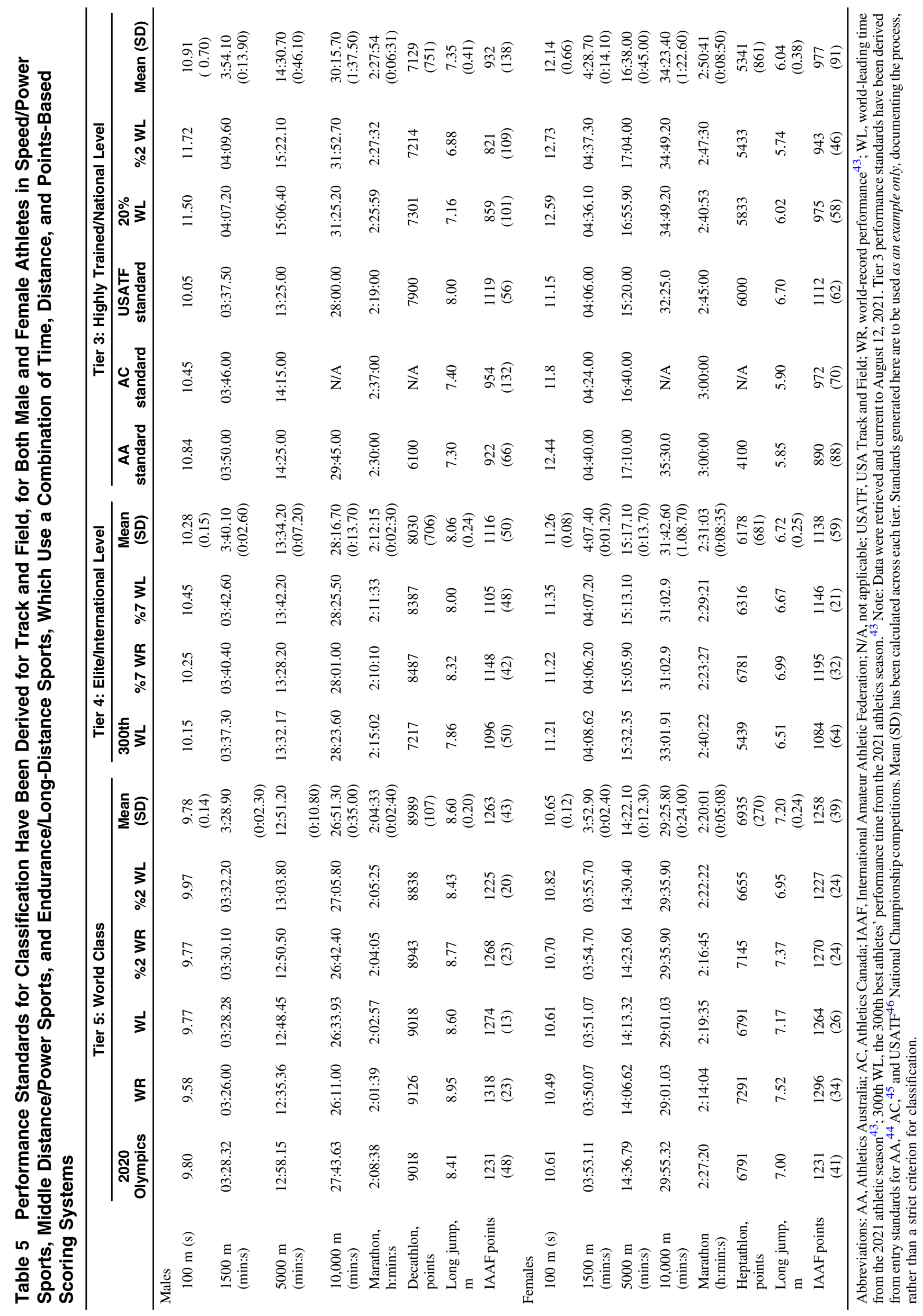




\section{Table 6 An Example Using Freestyle Snowboard (Big Air) of How the Participants Classification Framework Can Be Applied and Interpreted}

\begin{tabular}{|c|c|}
\hline Tier & Criteria for classification \\
\hline Tier 5: World Class & $\begin{array}{l}\text { - Performance achievements include: } \\
\text { (1) Medalist (top 3) at a major international event.* } \\
\text { - Athlete will be ranked within the Top } 10 \text { in World Rankings.** } \\
\text { - Athlete would be characterized as full-time athletes and are globally considered the top athletes in their sport. } \\
\text { *The authors consider "Major" international events to be those events that have a 900- or 1000-point WSPL weighting } \\
\text { for first place. These tend to include World Championships, Olympic Games, X Games, and FIS World Cups, which } \\
\text { have a strong, competitive field. } \\
\text { ** World Rankings should be determined from the WSPL, not the World Cup (FIS) ranking, as WSPL provides a } \\
\text { weighting to event based on field size and depth of field. Furthermore, WSLP include events that are of high-level } \\
\text { competition (eg, X Games), which are not included in the FIS rankings. }\end{array}$ \\
\hline $\begin{array}{l}\text { Tier 4: } \\
\text { Elite/International } \\
\text { Level }\end{array}$ & $\begin{array}{l}\text { - Performance achievements include: } \\
\text { (1) World Snowboard Points List ranking of top } 30 \text {. } \\
\text { (2) Finalist (top } 8 \text { and/or top third within a field) at a major event. } \\
\text { (3) Medalist/top } 3 \text { at continental-level or international events. *** } \\
\text { - Athlete is likely a National Team member. } \\
\text { - Competition-relevant tricks (competition-relevant difficulty) within } 7 \% \text { of world leading (for amplitude, execution) in } \\
\text { TWO spinning directions. } \\
\text { *** Continental events refer to events promoted as part of a continental "tour," for example, North American ("Nor- } \\
\text { Am") or European Cup events, and International events with } 700-800 \text { WSPL weighting. }\end{array}$ \\
\hline $\begin{array}{l}\text { Tier 3: Highly Trained/ } \\
\text { National Level }\end{array}$ & $\begin{array}{l}\text { - Performance achievements include: } \\
\text { (1) Medalist (top 3) at national events and/or championships. } \\
\text { (2) Finalist (top 8) at events continental-level international events. } \\
\text { - Athlete may be a member of a National Development Team or a top club, state, or provincial team athlete in countries } \\
\text { with substantial depth in the discipline. } \\
\text { - Competition-relevant tricks (competition-relevant difficulty) within } 20 \% \text { of world leading (for amplitude, execution) } \\
\text { in TWO spinning directions. }\end{array}$ \\
\hline
\end{tabular}

Tier 2: Trained/ Developmental

No performance standard required. Individuals must meet other criteria set out in Table 1.

Abbreviations: FIS, Fédération Internationale de Ski; WSPL, World Snowboards Points List. Note: Key considerations are based around participation in specific competitions and world rankings.

$38 \%$ of the gold medal-winning score. ${ }^{50}$ Accordingly, we suggest authors use their own knowledge of their sport to account for these differences, particularly when distinguishing between the top tiers within the Participant Classification Framework. An example of this can be seen when comparing track and field (Table 5) and snowboard (Table 6). In track and field, we limited Tier 5: World Class to athletes who qualify for the final of a major competition (the top approximately 8-15 athletes), with Tier 4: Elite/International Level including the top $\sim 300$ athletes. Alternatively, in Snowboard Tier 5: World Class are the medal-winning (top 3) athletes at major events, whereas the top 30 athletes are considered Tier 4: Elite/International Level. These sport-specific adjustments ensure that the quality of athletes included within a tier is not diluted by smaller field sizes, nor are exceptional athletes misclassified because of the larger number of high-performing athletes within an event.

Nationality Differences. In addition to differences between global participation rates, some sports are identified by marked pockets of success associated with specific countries or origins. Since the 1960s, East African countries, particularly Kenya and Ethiopia have dominated the sport of endurance running. ${ }^{43}$ Some of the factors suggested to explain their dominance in the sport include favorable anthropometric qualities, which led to biomechanical and metabolic advantages, a solid training foundation partly due to running often used as a form of transportation from a young age, hematological advantages resulting from living at moderate altitude, and a high motivation to succeed for the purpose of improving their socioeconomic status. ${ }^{51}$ Australia represents another example of concentrated sport success, with $60 \%$ of medals won at Olympic Games by Australians involving sports associated with water, whereas just $2.7 \%$ have come from Winter Sports. ${ }^{52}$ This is likely the result of a hot climate and extensive coastline, where sports associated with water are culturally valued and have high participation rates. There are numerous other examples of countries excelling at particular sports: Chinese athletes have dominated the sport of table tennis, winning $52 \%$ of all available Olympic medals in the sport, while US men's "dream team" in Basketball have won 16/20 Olympic gold medals. ${ }^{52}$ These region-specific differences in the success of a sport make global classification using the Participant Classification Framework challenging, particularly at the level of Tier 3: Highly Trained/National Level. This is evident in Table 5, where National Track-and-Field Championship entry standards from 3 different countries have been sourced. Here, performance standards for participation at the national level is higher in the United States, compared to Canada, despite being higher than countries with smaller populations such as Australia. To overcome these limitations, we suggest that world ranking and/or world-leading performance metrics be used in consultation with country-specific metrics (such as competition entry standards and national rankings) to gain an understanding of how an individual ranks in a global context, rather than within a strong or weak national field.

Gender Parity Within Sport. Finally, while female representation in sport has increased in recent years, gender parity is yet to 
be achieved, even in the most developed nations. On a global scale, females represented $49 \%$ and $41 \%$ of total competitors at the Tokyo 2020 Olympic and Paralympic Games, respectively, a vast improvement toward equality from the $38 \%$ and $26 \%$ who competed at Sydney in $2020 .{ }^{50}$ As with the introduction of any new event, it takes time to build depth within the field to see the best of human performance. Therefore, direct comparisons of male and female events within a sport may not be appropriate. For instance, although the men's 50-km race walk was included as an Olympic Event from 1932 to 2020, the women's event has never featured on the Olympic Games program, and since World Athletics started recording times in 2017, only 182 athletes have posted verified times. ${ }^{43}$ Such considerations should be included within the Participant Classification Framework, with different methods of benchmarking performance standards and world ranking brackets potentially required for males and females in some sports until complete gender parity, including participation opportunities, financial assistance, and performance potential is established.

\section{Chronological Age-The Junior Athlete Versus the Masters Athlete}

Age plays a role in sports performance since it can interact with the biological maturity of the young athlete, training, and competition maturity across the athlete's development pathway, and the biological decline associated with aging. However, these effects are not uniform across sports and even within sports; there are examples of extraordinary individuals who may achieve world-class status at a relatively early age, even in sports where intensive training loads commence in childhood (eg, swimming and gymnastics). Conversely, there are athletes who can remain internationally competitive in their late 30 s and 40s (eg, road cycling and long-distance triathlon). Since there are complex nuances between and within sports in relation to junior athletes and masters/veteran competitors, the first iteration of the Participant Classification Framework will focus on absolute performance rankings that are untroubled by age classifications. Following feedback and experience in the implementation of the Framework, future versions should consider age categories, or at least the inclusion of broad categories such as junior and masters competition.

\section{The Paralympic Athlete}

Despite the growing interest in Paralympic sport, the use and consistency for classifying training status remains in its infancy when compared to nondisabled sport. It's even more complex, since at International Paralympic Committee (IPC) sanctioned events, the primary diagnosis of a participant must be stable and belong to one of the 10 impairment types ( 8 physical impairments, as well as visual and intellectual impairments). This results in a heterogeneous population if simply described as a cohort of Para athletes. Many researchers have undertaken studies of Para athletes according to impairment type. For example, a series of studies examined respiratory mechanics during exercise that occurred in athletes with a cervical spinal cord injury. ${ }^{53,54}$ Likewise, studies specific to a sport where only one impairment type occurs, such as Cerebral Palsy football ${ }^{55}$ or goalball, which is a sport that involves players with a visual impairment, ${ }^{56}$ can be easier to categorize. However, within team sports such as wheelchair basketball, sports classification is based on the players' functional capacity to complete the skills necessary to play the sport, and players are allocated points. With the potential for such a diverse population within a sporting cohort, we suggest reporting the impairment type of each participant as this will help aid study interpretation. However, it should be considered that the disclosure of too many specific individual characteristics, such as age, onset of injury, and impairment classification may pose ethical issues with maintaining the anonymity of participants.

Within the context of the Participant Classification Framework, there are a number of factors which add an additional layer of complexity to categorizing Para athletes, such as "physical literacy" and "sporting age," which can influence an individual's transition from Tier 1 to Tier 5. For example, opportunities to train and participate in wheelchair basketball occur at an earlier age with congenital disabilities, ${ }^{57}$ and it's unclear whether participants with acquired disabilities can transfer skills from previous nondisabled sporting experiences. As stated by Dehghansai et al, ${ }^{57}$ considering the nuances of disability (ie, types of disability, severity), examining the influence of disability factors during investigations will be critical for extending our understanding of athletes' training and developmental trajectories, and indeed with classification of athletic ability. Furthermore, it is very common within Para sport for individuals to remain internationally competitive in their late 30s and 40s. In fact, in the women's Para Va'a (water paddling sport) at the Tokyo 2020 Paralympic Games, ${ }^{58}$ the silver medal was won by a 51-year-old, an athlete that transitioned between multiple Paralympic sports. Moreover, many competitive hand cyclists have sustained a spinal cord injury later in life and compete internationally well into their 40s. ${ }^{59}$ This is commonplace in wheelchair rugby too, with a recent study reporting a mean participant age of 32 (7) years. ${ }^{60}$ Finally, the use of performance standards derived from world rankings or world-leading times may not be appropriate for many Para sports at the Elite/International level, due to exceptionally small field sizes (as discussed above in "Depth of Participation in the Sport" section) or ever changing disability classification protocols/systems. The depth within Para sports fluctuates greatly, which can be explained by the total number of athletes with the impairment type and/or the talent pathway programs and coaching support systems. For example, at the Tokyo 2020 Paralympic Games, the T34 women's 100-m track race equated to a $24 \%$ difference between first and eighth, whereas the men's T64 event over the same distance had a $6.3 \%$ differential. ${ }^{58}$ While the event is the same, the 2 different impairment classifications and modalities (wheelchair propulsion vs ambulant athletes running) resulted in varying levels of performance within the event. We recognize that the Participant Classification Framework has been designed with the classification of nondisabled participants at the forefront, and there are numerous complexities which will make the classification of disabled participants more challenging. We hope to see future versions of the Participant Classification Framework adapted for the primary purpose of classifying Para athletes as more quantitative and qualitative data is developed.

\section{Conclusion}

In this paper, we have presented a practical, easy to implement framework for classifying the activity level and athletic ability of an individual. We envision wide-spread use of this tool in exercise 
and sport science-based literature that will provide uniformity around how we describe and examine cohorts of participants hereon in. In this first iteration of the framework, we have produced guidelines for classification based on training volume/physical activity metrics, performance standards, and skill level. It should be noted that sport-specific examples produced within this manuscript should not be used as a rigid classification system; rather, these performance standards should be updated over time and evolve as the sport, and world-leading performances naturally progress. Furthermore, this framework has intentionally been created so that it can be easily adaptable to fit many different sports. We suggest authors adapt the framework to fit the cohort in question, paying particular attention to features such as the size and depth of the field of a sport, and a cohort's nationality and gender parity within the sport. Finally, we have highlighted issues we foresee when implementing this framework, particularly around special populations like junior/masters athletes and individuals with a disability (including Paralympic athletes). We would like to see future versions of this framework be specifically developed to target these populations.

\section{References}

1. McAuley AB, Baker J, Kelly AL. Defining "elite" status in sport: from chaos to clarity [published online ahead of print August 5, 2021]. Ger J Exerc Sport Res. doi:10.1007/s12662-021-00737-3

2. Swann C, Moran A, Piggott D. Defining elite athletes: issues in the study of expert performance in sport psychology. Psychol Sport Exerc. 2015;16:3-14. doi:10.1016/j.psychsport.2014.07.004

3. Decroix L, De Pauw K, Foster C, Meeusen R. Guidelines to classify female subject groups in sport-science research. Int J Sports Physiol Perform. 2016;11(2):204-213. PubMed ID: 26182438 doi:10.1123/ ijspp.2015-0153

4. De Pauw K, Roelands B, Cheung SS, De Geus B, Rietjens G, Meeusen R. Guidelines to classify subject groups in sport-science research. Int J Sports Physiol Perform. 2013;8(2):111-122. PubMed ID: 23428482 doi:10.1123/ijspp.8.2.111

5. Betts JA, Gonzalez JT, Burke LM, et al. PRESENT 2020: text expanding on the checklist for proper reporting of evidence in sport and exercise nutrition trials. Int J Sport Nutr Exerc Metab. 2020;30(1):213. PubMed ID: 31945740 doi:10.1123/ijsnem.2019-0326

6. Marquet L-A, Hausswirth C, Molle O, et al. Periodization of carbohydrate intake: short-term effect on performance. Nutrients. 2016;8(12):755. doi:10.3390/nu8120755

7. Marquet L-A, Brisswalter J, Louis J, et al. Enhanced endurance performance by periodization of CHO intake: "sleep low" strategy. Med Sci Sports Exerc. 2016;48(4):663-672. PubMed ID: 26741119 doi:10.1249/MSS.0000000000000823

8. Burke LM, Ross ML, Garvican-Lewis LA, et al. Low carbohydrate, high fat diet impairs exercise economy and negates the performance benefit from intensified training in elite race walkers. J Physiol. 2017; 595(9):2785-2807. PubMed ID: 28012184 doi:10.1113/JP273230

9. Gejl KD, Thams LB, Hansen M, et al. No superior adaptations to carbohydrate periodization in elite endurance athletes. Med Sci Sports Exerc. 2017;49(12):2486-2497. PubMed ID: 28723843 doi:10.1249/ MSS.0000000000001377

10. Burke LM, Sharma AP, Heikura IA, et al. Crisis of confidence averted: impairment of exercise economy and performance in elite race walkers by ketogenic low carbohydrate, high fat (LCHF) diet is reproducible. PLoS One. 2020;15(6):e0234027. PubMed ID: 32497061 doi:10.1371/journal.pone.0234027
11. Peeling P, Binnie MJ, Goods PS, Sim M, Burke LM. Evidence-based supplements for the enhancement of athletic performance. Int J Sport Nutr Exerc Metab. 2018;28(2):178-187. PubMed ID: 29465269 doi:10.1123/ijsnem.2017-0343

12. Jones AM. Dietary nitrate supplementation and exercise performance. Sports Med. 2014;44(suppl 1):35-45. doi:10.1007/s40279-014-0149-y

13. Burke LM, Hall R, Heikura IA, et al. Neither beetroot juice supplementation nor increased carbohydrate oxidation enhance economy of prolonged exercise in elite race walkers. Nutrients. 2021;13(8):2767. PubMed ID: 34444928 doi:10.3390/nu13082767

14. Ericsson KA, Krampe RT, Tesch-Römer C. The role of deliberate practice in the acquisition of expert performance. Psychol Rev. 1993;100(3):363. doi:10.1037/0033-295X.100.3.363

15. Gladwell M. Outliers: The Story of Success. Little, Brown and Company; 2008.

16. Lloyd RS, Oliver JL, Faigenbaum AD, Myer GD, Croix MBDS. Chronological age vs. biological maturation: implications for exercise programming in youth. J Strength Cond Res. 2014;28(5):1454-1464. PubMed ID: 24476778 doi:10.1519/JSC.0000000000000391

17. MacArthur DG, North KN. Genes and human elite athletic performance. Hum Genet. 2005;116(5):331-339. PubMed ID: 15726413 doi:10.1007/s00439-005-1261-8

18. Vaeyens R, Güllich A, Warr CR, Philippaerts R. Talent identification and promotion programmes of Olympic athletes. J Sports Sci. 2009;27(13):1367-1380. PubMed ID: 19787538 doi:10.1080/ 02640410903110974

19. Jeukendrup AE, Craig NP, Hawley JA. The bioenergetics of world class cycling. J Sci Med Sport. 2000;3(4):414-433. PubMed ID: 11235007 doi:10.1016/S1440-2440(00)80008-0

20. Bull FC, Al-Ansari SS, Biddle S, et al. World Health Organization 2020 guidelines on physical activity and sedentary behaviour. $\mathrm{Br} J$ Sports Med. 2020;54(24):1451-1462. PubMed ID: 33239350 doi:10. 1136/bjsports-2020-102955

21. Australian Institute of Health Welfare. Physical activity across the life stages. 2018. Accessed June 2021. https://www.aihw.gov.au/reports/ physical-activity/physical-activity-across-the-life-stages/contents/ table-of-contents

22. Centers for Disease Control and Prevention. National Center for Chronic Disease Prevention and Health Promotion, Division of Nutrition, Physical Activity, and Obesity: Data, trends and maps. Updated November 19, 2021. Accessed May 2021. https://www.cdc. gov/nccdphp/dnpao/data-trends-maps/index.html

23. Sport England. Active Lives Adult Survey November 2019/20 Report. Sport England. April 2021. Accessed June 2021.

24. Statistics Canada. Physical activity, self reported, adult, by age group. Updated September 2021. Accessed May 2021. https:// www150.statcan.gc.ca/t1/tbl1/en/tv.action?pid=1310009613

25. AusPlay. Australians' Participation and Involvement in Summer Olympic and Paralympic Sports. Sport Australia. 2021. Accessed June 2021. https://www.clearinghouseforsport.gov.au/research/ ausplay/results

26. Cooke K. 37\% of people in the UK never exercise or play sport. 2018. Accessed September 2021. https://www.kantar.com/uki/inspiration/sportleisure/37-per-cent-of-people-in-the-uk-never-exercise-or-play-sport/

27. Lange D. Physical activity—Statistics \& facts. 2021. Accessed September 2021. https://www.statista.com/topics/1749/physical-activity/

28. Hopkins WG. Measures of reliability in sports medicine and science. Sports Med. 2000;30(1):1-15. PubMed ID: 10907753 doi:10.2165/ 00007256-200030010-00001

29. World Anti-Doping Agency. Whereabouts. February 2009. Accessed August 2021. https://www.wada-ama.org/en/questions-answers/ whereabouts 
30. Jones AM. The physiology of the world record holder for the women's marathon. Int J Sports Sci Coach. 2006;1(2):101-116. doi:10.1260/174795406777641258

31. Carr AJ, Saunders PU, Garvican-Lewis LA, Vallance BS. Altitude and heat training in preparation for competitions in the heat: a case study. Int J Sports Physiol Perform. 2020;15(9):1344-1348. doi:10. 1123/ijspp.2019-0292

32. Schmitt L, Bouthiaux S, Millet GP. Eleven years' monitoring of the world's most successful male biathlete of the last decade. Int J Sports Physiol Perform. 2020;16(6):900-905. PubMed ID: 32887848 doi:10.1123/ijspp.2020-0148

33. @ dlhamilton82. Ever wonder why there are so few training studies in trained populations? September 9, 2016. Accessed July 2021. https:// twitter.com/dlhamilton82/status/774197423342252032

34. Jones AM, Kirby BS, Clark IE, et al. Physiological demands of running at 2-hour marathon race pace. J Appl Physiol. 2021;130(2): 369-379. PubMed ID: 33151776 doi:10.1152/japplphysiol.00647. 2020

35. Eaton TR, Potter A, Billaut F, et al. A combination of amino acids and caffeine enhances sprint running capacity in a hot, hypoxic environment. Int J Sport Nutr Exerc Metab. 2016;26(1):33-45. PubMed ID: 26248498 doi:10.1123/ijsnem.2015-0108

36. Muggeridge DJ, Sculthorpe N, James PE, Easton C. The effects of dietary nitrate supplementation on the adaptations to sprint interval training in previously untrained males. J Sci Med Sport. 2017;20(1): 92-97. PubMed ID: 27260004 doi:10.1016/j.jsams.2016.04.014

37. Niemiro GM, Edwards T, Barfield J, et al. Circulating progenitor cell response to exercise in wheelchair racing athletes. Med Sci Sports Exerc. 2018;50(1):88-97. PubMed ID: 28806276 doi:10.1249/MSS. 0000000000001402

38. Baumgart JK, Brurok B, Sandbakk $\varnothing$. Peak oxygen uptake in Paralympic sitting sports: a systematic literature review, meta-and pooled-data analysis. PLoS One. 2018;13(2):e0192903. PubMed ID: 29474386 doi:10.1371/journal.pone.0192903

39. Calderbank JA, Comfort P, McMahon JJ. Association of jumping ability and maximum strength with dive distance in swimmers. Int $J$ Sports Physiol Perform. 2020;16(2):296-303. PubMed ID: 33276324 doi:10.1123/ijspp.2019-0773

40. Stefani RT. A taxonomy of sports rating systems. IEEE Trans Syst Man Cybern Part A Syst Humans. 1999;29(1):116-120. doi:10.1109/ 3468.736367

41. Spotrac. Sports contracts, salaries, caps, bonuses \& transactions. 2021. Accessed September 2021. https://www.spotrac.com/

42. DiFiori JP, Güllich A, Brenner JS, et al. The NBA and youth basketball: recommendations for promoting a healthy and positive experience. Sports Med. 2018;48(9):2053-2065. PubMed ID: 29961207 doi:10.1007/s40279-018-0950-0

43. World Athletics. All time top lists. 2021. Accessed September 2021. https://www.worldathletics.org/records/all-time-toplists/

44. Athletics Australia. 2021-22 Australian Championship Entry Standards. March 2021. Accessed July 2021. https://www.athletics.com. au/entrystandards/

45. Athletics Canada. 2019 Canadian Track and Field Champsionships. 2019. Accessed July 2021.https://athletics.ca/events/montreal2019/
46. USA Track and Field. Qualifying standards. 2020. Accessed July 2021. https://www.usatf.org/events/2021/2020-u-s-olympic-teamtrials-track-field/qualifying-standards

47. Weather Underground. Sapporo, Hokkaido, Japan Weather Conditions. 2021. Accessed September 2021. https://www.wunderground. $\mathrm{com} /$ weather/jp/sapporo

48. Association of Tennis Professionals. 2021 ATP Official Rule Book. Chapter 9: FedEx ATP Rankings. January 2021. Accessed September 2021. https://www.atptour.com/en/corporate/rulebook

49. Internation Judo Federation. IJF WRL Event Points. 2021. Accessed September 2021. https://www.ijf.org/wrl_downloads

50. International Olympic Committee. Olympic schedule \& results. 2021. Accessed September 2021. https://olympics.com/tokyo-2020/olympicgames/en/results/all-sports/olympic-schedule.htm

51. Wilber RL, Pitsiladis YP. Kenyan and Ethiopian distance runners: what makes them so good? Int J Sports Physiol Perform. 2012; 7(2):92-102. PubMed ID: 22634972 doi:10.1123/ijspp.7.2.92

52. Olympedia. Countries. November 2018. Accessed August 2021. https://www.olympedia.org/countries

53. West CR, Goosey-Tolfrey VL, Campbell IG, Romer LM. Effect of abdominal binding on respiratory mechanics during exercise in athletes with cervical spinal cord injury. J Appl Physiol. 2014; 117(1):36-45. PubMed ID: 24855136 doi:10.1152/japplphysiol. 00218.2014

54. West CR, Campbell IG, Goosey-Tolfrey VL, Mason BS, Romer LM. Effects of abdominal binding on field-based exercise responses in Paralympic athletes with cervical spinal cord injury. J Sci Med Sport. 2014;17(4):351-355. PubMed ID: 23880254 doi:10.1016/j.jsams. 2013.06.001

55. Yanci J, Castagna C, Los Arcos A, et al. Muscle strength and anaerobic performance in football players with cerebral palsy. Disabil Health J. 2016;9(2):313-319. PubMed ID: 26723466 doi:10.1016/j. dhjo.2015.11.003

56. Gawlik K, Zwierzchowska A, Rosołek B. Evaluation of lipid metabolism and nutritional status in male goalball players. J Hum Kinet. 2015;48(1):141-147. PubMed ID: 26834882 doi:10.1515/hukin2015-0100

57. Dehghansai N, Lemez S, Wattie N, Baker J. Training and development of Canadian wheelchair basketball players. Eur J Sport Sci. 2017;17(5):511-518. PubMed ID: 28099092 doi:10.1080/17461391. 2016.1276636

58. International Paralympic Committee. Tokyo 2020 Results. 2021. Accessed September 2021. https://www.paralympic.org/tokyo-2020/ results

59. Graham-Paulson T, Perret C, Goosey-Tolfrey V. Case study: dose response of caffeine on $20-\mathrm{km}$ handcycling time trial performance in a paratriathlete. Int J Sport Nutr Exerc Metab. 2018;28(3):274-278. PubMed ID: 29091469 doi:10.1123/ ijsnem.2017-0089

60. Rhodes JM, Mason BS, Perrat B, Smith MJ, Malone LA, Goosey-Tolfrey VL. Activity profiles of elite wheelchair rugby players during competition. Int J Sports Physiol Perform. 2015; 10(3):318-324. PubMed ID: 25202822 doi:10.1123/ijspp.20140203 